\title{
Bem1p, a scaffold signaling protein, mediates cyclin-dependent control of vacuolar homeostasis in Saccharomyces cerevisiae
}

\author{
Bong-Kwan Han, ${ }^{1}$ Lydia M. Bogomolnaya, ${ }^{1}$ James $M$. Totten, ${ }^{1}$ Heidi M. Blank, ${ }^{1}$ \\ Lawrence J. Dangott, ${ }^{1,2}$ and Michael Polymenis ${ }^{1,3}$ \\ ${ }^{1}$ Department of Biochemistry and Biophysics, and ${ }^{2}$ The Protein Chemistry Laboratory, Texas A\&M University, \\ College Station, Texas 77843, USA
}

\begin{abstract}
How proliferating cells maintain the copy number and overall size of their organelles is not clear. We had previously reported that in the budding yeast Saccharomyces cerevisiae the G1 cyclin Cln3p is required for vacuolar (lysosomal) homotypic fusion and loss of $\mathrm{Cln} 3 p$ leads to vacuolar fragmentation. The Cdc42p GTPase is also required for vacuole fusion. Here we show that the scaffold protein Bem1p, a critical regulator of Cdc42p activity, is a downstream effector of $\mathrm{Cln} 3 \mathrm{p}$ and the cyclin-dependent kinase (Cdk) Cdc28p. Our results suggest that Bem1p is phosphorylated in a Cdk-dependent manner to promote vacuole fusion. Replacing Ser72 with Asp, to mimic phosphorylation at an optimal Cdk-consensus site located in the first SH3 domain of Bem1p, suppressed vacuolar fragmentation in cells lacking Cln3p. Using in vivo and in vitro assays, we found that $C \ln 3 p$ was unable to promote vacuole fusion in the absence of Bem1p or in the presence of a nonphosphorylatable Bem1p-Ser72Ala mutant. Furthermore, activation of Cdc42p also suppressed vacuolar fragmentation in the absence of $\mathrm{Cln} 3 \mathrm{p}$. Our results provide a mechanism that links cyclin-dependent kinase activity with vacuole fusion through Bem1p and the Cdc42p GTPase cycle.
\end{abstract}

[Keywords: Vacuole; CLN; CDC42; BEM1; cell size; START]

Received August 5, 2005; revised version accepted August 30, 2005.

Overall cell size and macromolecular composition remain unaffected after successive rounds of cell division. This phenomenon also extends to intracellular membrane-bound organelles, because the copy number and size of any given organelle compartment remain constant in dividing cells (Warren and Wickner 1996; Shorter and Warren 2002). Implicit in this central aspect of cellular physiology is a tight coordination between cell division and organelle biogenesis, but in most cases the mechanisms remain mysterious (Warren and Wickner 1996; Shorter and Warren 2002).

Vacuoles in Saccharomyces cerevisiae serve as repositories of metabolites and low molecular weight compounds and are analogous to the lysosomes of animal cells, containing numerous hydrolases (Roberts et al. 1991; Jones et al. 1997). The vacuole is a low-copy organelle, and yeast cells typically contain one to three vacuoles. The large size of the vacuolar compartment $(\sim 25 \%$ of the total cellular volume) (Wiemken and Durr

${ }^{3}$ Corresponding author.

E-MAIL polymenis@tamu.edu; FAX (979) 845-9274.

Article published online ahead of print. Article and publication date are at http://www.genesdev.org/cgi/doi/10.1101/gad.1361505.
1974) and the availability of vacuole-specific vital fluorescent dyes facilitate observations of overall vacuolar morphology. Defects in self (homotypic) fusion of vacuolar vesicles lead to vacuolar fragmentation (Seeley et al. 2002). Thus, homotypic fusion is very important for vacuolar homeostasis and it can also be evaluated in vitro (Wickner and Haas 2000). Although daughter cells of certain vacuolar inheritance mutants can be born without a vacuole, they must form a new one before they can pass through a point in late G1 called START and initiate DNA replication and a new round of cell division (Weisman 2003). A recent report also suggested that in Candida albicans the vacuolar compartment may impact on cell cycle progression and hyphal development (Barelle et al. 2003). Nonetheless, it is not known how the molecular machinery that regulates cell cycle progression also affects vacuolar biogenesis or vice versa.

In late G1 START completion is mediated by Cdc $28 \mathrm{p}$ (a cyclin-dependent kinase [Cdk]) in association with one of the G1 cyclins, Cln1,2,3p. Cells lacking all three $C L N$ genes are inviable and cannot complete START (Richardson et al. 1989). Cln3p functions upstream of $C \ln 1,2 p$ activating the G1/S transcription program (Dirick et al. 1995), where $\sim 200$ genes (CLN1,2 among them) are tran- 
scribed (Spellman et al. 1998). Cln3p/Cdc28p phosphorylates Whi5p, a repressor of the G1/S transcription factor $\mathrm{SBF}$, thereby releasing Whi5p from SBF and activating START transcription (Costanzo et al. 2004; de Bruin et al. 2004; Schaefer and Breeden 2004). In addition, our earlier findings provided evidence for a novel function of $C \ln 3 p$ in vacuolar homotypic fusion, separate from its role in G1/S transcription and not shared by other G1 cyclins (Han et al. 2003).

A central polarity-establishment factor in a variety of organisms (from yeast to humans) is Cdc42p, a Rho-type small GTPase that orchestrates numerous processes necessary for polarization, such as septin and actin organization and membrane trafficking, in response to cell cycle transitions and environmental cues (EtienneManneville 2004; Irazoqui and Lew 2004). Cdc42p membrane localization is not restricted to the plasma membrane but includes internal membranes, notably vacuolar membranes (Richman et al. 2004). Furthermore, Cdc $42 p$ is one of several GTPases required for vacuolar homotypic fusion (Eitzen et al. 2001; Muller et al. 2001). Reorganization of vacuole-bound actin is needed for vacuolar homotypic fusion, and proteins of the Cdc42p-dependent processes necessary for actin remodeling are enriched on vacuolar membranes (Eitzen et al. 2002). Among numerous Cdc42p effectors and interacting proteins, the scaffold protein Bemlp is critical for proper Cdc42p activation (Irazoqui et al. 2003). After the $\mathrm{Cln} 3 \mathrm{p} / \mathrm{Cdc} 28 \mathrm{p}-\mathrm{mediated}$ initiation of the G1/S transcription program, the burst of late G1-phase Cdk activity involving Cln1,2p/Cdc28p and Pcl1,2p/Pho85p (another cyclin/Cdk complex) triggers a pathway that leads to phosphorylation of Cdc24p. Cdc24p is a Cdc42p guanine nucleotide exchange factor (GEF). Once at the bud site Cdc24p binds Bem 1p, and Cdc42p-dependent actin reorganization necessary for bud emergence takes place (Gulli et al. 2000; Bose et al. 2001; Moffat and Andrews 2004).

In this report we show that Bem $1 p$ is required for vacuolar homotypic fusion and that the overall vacuolar compartment in bem1s cells is enlarged but fragmented, similar to $\operatorname{cln} 3 \Delta$ cells. Furthermore, Bem1p is phosphorylated in a Cln3p-dependent manner at Ser72. A single S72D substitution in Bemlp (which mimics phosphorylation) or overexpression of $C D C 42$ or $C D C 24$ suppresses the vacuolar fragmentation of $\operatorname{cln} 3 \Delta$ cells. Conversely, substituting Ser72 in Bem1p with Ala blocks the ability of $\mathrm{Cln} 3 \mathrm{p}$ to promote vacuole fusion, in vivo and in vitro. The results we report here suggest that $C \ln 3 p$ impacts on vacuolar homeostasis through Bemlp and Cdc42p.

\section{Results}

Cln3p is required for an early step of vacuolar homotypic fusion

We had previously shown that $C \ln 3 p$ is required for vacuolar homotypic fusion (Han et al. 2003). Vacuolar homotypic fusion is composed of several distinct se- quential steps: priming, docking, and fusion (Wickner and Haas 2000). To study the kinetics of the reaction, order of addition experiments can be done, whereby the fusion reaction progressively becomes resistant to the addition of various inhibitors, once the inhibitor-sensitive step has been completed. For example, placement on ice blocks all steps and thus results in a "late-stage" inhibition profile, which is also similar to the GTP- $\gamma$-S inhibition profile. There are several GTPases involved in more than one step, including the last fusion step, which leads to a late-stage inhibition profile by GTP- $\gamma$-S (Eitzen et al. 2000, 2001). On the other hand, the ionophore FCCP blocks the docking step and it does not significantly inhibit the overall reaction if added late.

Using this approach, we decided to determine the stage of the overall reaction that requires $C \ln 3 p$ for its completion, with cytosol from cells expressing HAtagged $C \ln 3 p$. We then added an anti-HA antibody as an inhibitor at various time points during the reaction and measured the overall fusion activity at the end of the 90-min incubation period (Fig. 1). This experiment was

A

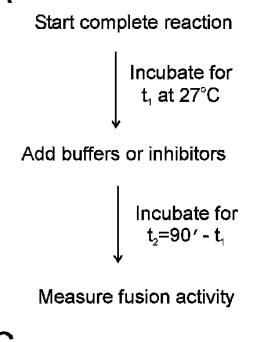

B

C
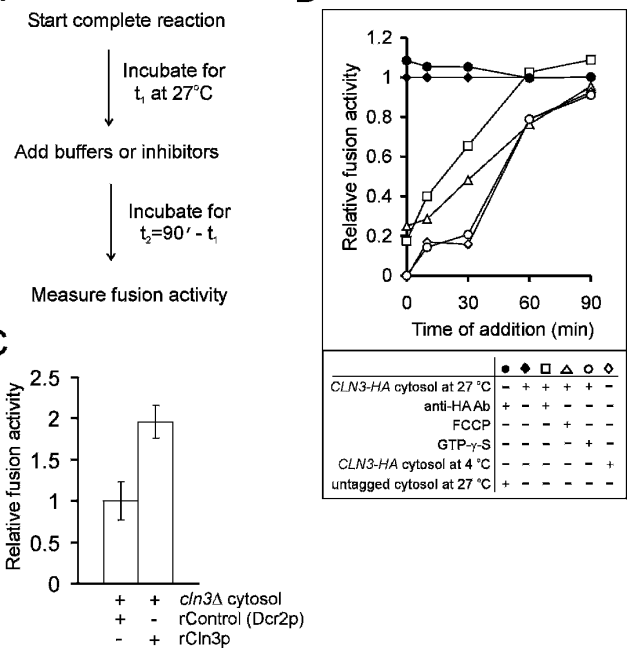

Figure 1. $C \ln 3 p$ is required for an early step during vacuole fusion. (A) A schematic of the experimental strategy is shown. $(B)$ Fusion reactions were incubated at $27^{\circ} \mathrm{C}$ with cytosol from a CLN3-HA strain. At the indicated times of addition $\left(\mathrm{t}_{1}\right)$ shown on the $X$-axis, aliquots were removed and added to tubes containing anti-HA Ab, FCCP, GTP- $\gamma-\mathrm{S}$, or buffer and incubated at $27^{\circ} \mathrm{C}$. The activity from the buffer-containing tube at $27^{\circ} \mathrm{C}$ was set as $100 \%$ fusion activity. Another buffer-containing tube was incubated on ice and its activity was set at $0 \%$ fusion activity. As a control, aliquots of reactions with cytosol from the untagged isogenic strain were also added at various time points to tubes containing the anti-HA $\mathrm{Ab}(\bullet)$ or buffer, and treated in the same manner as above. Reactions were incubated for a total of $90 \mathrm{~min}$ and then assayed for alkaline phosphatase, the relative values of which are shown on the $Y$-axis. $(C)$ Fusion reactions were performed as described in Materials and Methods, using cytosol from $c \ln 3 \Delta$ cells and recombinant proteins from bacteria as indicated. Fusion was evaluated colorimetrically and the average and standard deviation of the relative fusion activities from at least three independent experiments is shown. 
done in parallel with several other reactions, to compare the profile of Cln3p withdrawal to the profiles of known fusion inhibitors (Fig. 1). From this analysis, it appears that the reaction becomes resistant to removal of $C \ln 3 p$ with kinetics slightly earlier than the kinetics for FCCP addition (Fig. 1). Thus, once docking is complete, Cln3p is not required for vacuolar homotypic fusion.

To further test whether the requirement for $\mathrm{Cln} 3 \mathrm{p}$ in vacuole fusion is direct, and not somehow due to indirect effects resulting from Cln3p's role in G1/S transcription, we performed the in vitro fusion reaction using cytosol from cln3s cells, supplemented with Cln3p expressed in bacteria (Fig. 1C). Addition of recombinant $\mathrm{Cln} 3 \mathrm{p}$, but not of the unrelated control protein Dcr2p (Pathak et al. 2004), increased fusion activity (Fig. 1C). These results argue that there is a direct requirement for $\mathrm{Cln} 3 \mathrm{p}$ in vacuole fusion.

Bem1p impacts on vacuolar biogenesis, downstream of $C \ln 3 p$

To understand Cln3p's vacuolar function, we reasoned that one of the numerous known regulators of homotypic fusion might be targeted by Cln3p. Possible downstream effectors of $\mathrm{Cln} 3 \mathrm{p} / \mathrm{Cdc} 28 \mathrm{p}$ in vacuolar homotypic fusion may display the following properties: Loss-offunction mutations should lead to vacuolar and overall cell size enlargement, loss-of-function mutations should lead to vacuolar fragmentation, and the putative effectors should be Cdk targets. Recent genome-wide studies focused on these properties, namely, altered cell size (Jorgensen et al. 2002; Zhang et al. 2002), fragmented vacuolar morphology (Seeley et al. 2002), and proteins that are phosphorylated by Cdc28p in whole-cell extracts (Ubersax et al. 2003). There are $>100$ gene products in each data set (Fig. 2A). For example, there were 181 proteins that were significantly phosphorylated by Clb2p/ Cdc28p (with a $P$-score $\geq 2$, which is the logarithm of the amount of phosphate incorporated per nanogram of protein). Yet, only Cln3p itself and Bem $1 p$ were present in all three data sets (Fig. 2A).

Using the in vitro fusion assay, we found that reactions with cytosol from BEM1-TAP cells in the presence of inhibitory rabbit IgG (which targets the TAP domain) had significantly reduced fusion activity (Fig. 2B). Loss of Bem $1 p$ or Cln3p also leads to vacuolar fragmentation in $\sim 40-60 \%$ of the cells (see Fig. 3A,B; Seeley et al. 2002; Han et al. 2003). Furthermore, cells lacking BEM1 were larger overall $(\sim 40 \%)$ (Figs. 2, 3) than wild-type cells and, importantly, their vacuolar compartment was also disproportionately enlarged (about twofold) (Figs. 2, 3). Finally, Cdc28p readily phosphorylated Bem $1 \mathrm{p}$ $(P$-score $=3.4)$ in the phosphorylation assays by Ubersax et al. (2003). Consequently, we decided to evaluate Bem $1 \mathrm{p}$ as a putative $\mathrm{Cln} 3 \mathrm{p} / \mathrm{Cdc} 28 \mathrm{p}$ effector in vacuolar biogenesis.

To test whether $C \ln 3 p$ might mediate its effects in vacuolar biogenesis through Bemlp, we measured the overall cell size and vacuolar size of CLN3 and BEM1
A

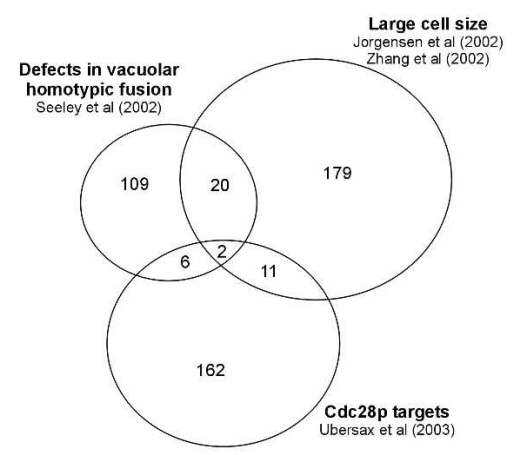

B

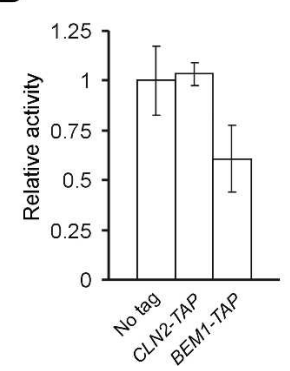

C

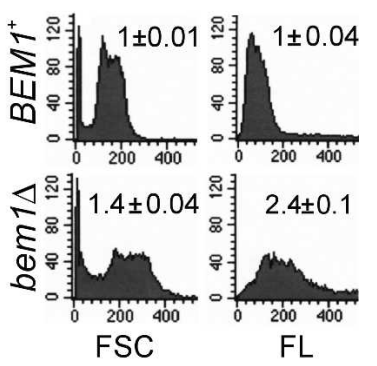

Figure 2. Bemlp is required for vacuole fusion and vacuolar size homeostasis. (A) Venn diagrams from the indicated genome-wide studies; the number of genes corresponding to different phenotypes in each case is shown. $(B)$ Vacuole fusion using cytosol from untagged, CLN2-TAP-tagged, or BEM1-TAPtagged strains (see Table 1), carried out in the presence of rabbit IgG antibody. Each measurement was evaluated upon addition of rabbit IgG or buffer. The buffer-containing reactions were then incubated at $27^{\circ} \mathrm{C}$ or on ice (setting the $100 \%$ and $0 \%$ values of fusion activity). The IgG-containing reactions were incubated at $27^{\circ} \mathrm{C}$. Fusion was evaluated colorimetrically and the average and standard deviation from at least three independent experiments is shown. $(C)$ Cell and vacuole size of live CDCFDA-stained $B E M 1^{+}$and bem1s cells (in the diploid BY4743 background) was evaluated by flow cytometry. The number of cells is shown on the $Y$-axis, and the $X$-axis indicates forward angle scattering, which is indicative of cell size (FSC), or vacuolar fluorescence (FL). The average and standard deviation from five independent measurements in each case is shown.

mutant combinations and also microscopically examined their vacuolar morphology. Loss of Cln3p or Bem $1 p$ leads to cellular and vacuolar enlargement and vacuolar fragmentation (Fig. 3). Conversely, cells expressing a stabilized form of $\mathrm{Cln} 3 \mathrm{p}$ from the dominant gain-offunction $C L N 3-2^{D}$ allele are smaller overall (Cross 1988), and their vacuolar compartment is also smaller (see Fig. 3A; Han et al. 2003). However, the CLN3-2 ${ }^{D}$ allele was unable to reduce cell and vacuole size in the absence of Bem1p (Fig. 3A). Furthermore, combined loss of Bem1p and $C \ln 3 p$ did not lead to an additive cellular or vacuolar enlargement or vacuolar fragmentation (Fig. 3B). These results are consistent with the idea that Bemlp is required for Cln3p's effects on cell and vacuole size, and that Bem $1 p$ and $C \ln 3 p$ do not affect vacuolar biogenesis through separate independent pathways. 
A

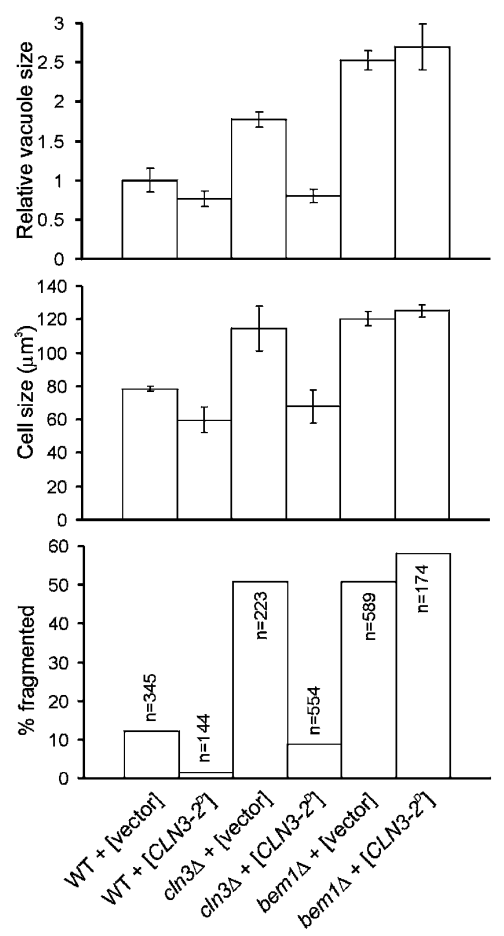

B
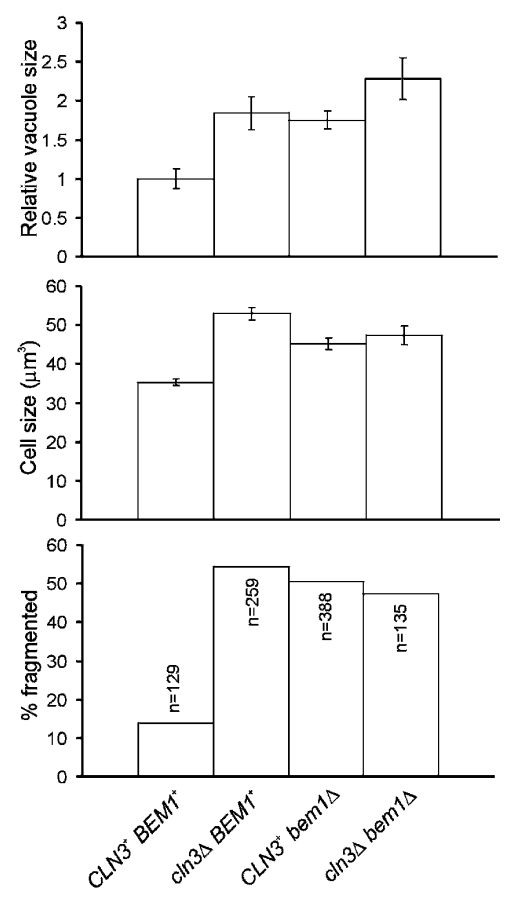

Figure 3. $C \ln 3 p$ requires Bem $1 p$ to affect cell and vacuole size and vacuolar morphology. (A) The relative vacuole size of the indicated strains (all in the diploid BY4743 background) was measured as in Figure 2, and cell size was measured with a channelyzer. The average and standard deviation from four independent measurements in each case is shown. The indicated strains were also stained with FM4-64 to observe their vacuolar morphology by fluorescence microscopy. The percent of cells with vacuolar fragmentation and the number of cells scored in each case $(n)$ is shown. $(B)$ The indicated parameters were measured as in $A$, using the strains shown (in the haploid BY4741 background).

\section{Bem1p is phosphorylated in a Cdk-dependent manner}

It has been previously mentioned in an earlier report that Bem 1p might be a phosphoprotein, because phosphatase treatment leads to faster migration of Bem1p during SDS-PAGE (Leeuw et al. 1995). Indeed, immunoprecipitated TAP-tagged Bem $1 \mathrm{p}$ migrated faster upon phosphatase treatment (Fig. 4A). Bemlp has four potential Cdk phosphorylation sites: two [ST]-P-X-[KR] sites at Thr51 and Ser72 and two [ST]-P sites at Thr26 and Ser60, respectively. As a first step toward establishing possible Cdk-mediated phosphorylation events, we asked whether an antibody directed against phosphorylated [ST]-P recognized Bem $1 \mathrm{p}$ immunoprecipitated from cell extracts. From these cell extracts we sequentially immunoprecipitated Bem1p-TAP using IgG and calmodulin beads (Fig. 4B). The immunoprecipitated protein was readily recognized by the PAP reagent, via the protein $\mathrm{A}$ domain (Fig. 4B). To use the anti-phospho-[ST]-P antibody, after immunoprecipitations with IgG beads, we cleaved the immunoprecipitated product with TEV protease to remove the protein A part of the TAP epitope. Immunoblotting with the anti-phospho-[ST]-P antibody recognized the cleaved Bem1p-CBP (which now lacks the protein A part of the TAP tag) (Fig. 4B). Recognition by the anti-phospho-[ST]-P antibody was phosphorylation dependent, because the signal was significantly reduced after treatment with phosphatase (Fig. 4C). When normalized for Bem1p levels using an antibody that recognizes the remaining part of the TAP tag after TEV cleavage, treatment with phosphatase removed $>70 \%$ of the signal (Fig. 4C, bottom).
To further evaluate whether Cdc28p activity contributes to Bem 1p phosphorylation, we used cdc28-as 1 cells, which express an engineered version of Cdc28p that is inhibited by the ATP analog 1NM-PP1 (Bishop et al. 2000). These cells were then transformed with a lowcopy centromeric plasmid carrying cMyc-tagged Bem $1 \mathrm{p}$ (Irazoqui et al. 2003). Upon treatment with the inhibitory drug, the mobility of Myc-tagged Bemlp increased on SDS-PAGE, consistent with dephosphorylation (Fig. 4D). No such effects were seen upon treatment with DMSO or in the isogenic strain that does not carry the engineered cdc28-as1 allele (Fig. 4D). We had previously reported that shifting temperature-sensitive cdc28-1 cells to their nonpermissive temperature led to vacuolar fragmentation (Han et al. 2003). To further confirm that Cdc28p-associated kinase activity is required for vacuole homotypic fusion, we performed the in vitro fusion reaction using cytosol from cdc28-as 1 cells, and we found that upon treatment with the inhibitory drug, fusion activity was reduced to $\sim 40 \%$ of the activity when DMSO alone was added (Fig. 4E). Together, all of the above results support the hypothesis that Bem $1 p$ is phosphorylated in a Cdc28p-dependent manner in vivo.

\section{Ser72 is critical for Bem1p's role in vacuolar homeostasis}

We then examined whether the Bemlp potential Cdk phosphorylation sites are conserved in other Saccharomyces species (Cliften et al. 2003; Kellis et al. 2003) and in a couple of Candida species for which genome infor- 
Figure 4. Bemlp might be phosphorylated in a Cdkdependent manner. (A) Schematic of the TAP-tagged Bem 1 p. Bem lp-TAP was isolated from cells carrying a chromosomal TAP-tagged BEM1 copy (BEM1-TAP, strain 7499374) (see Table 1) by immunoprecipitation using IgG and treated with calf intestinal phosphatase (CIP) as indicated. The samples were then processed for SDS-PAGE and immunoblotting with the PAP reagent. (B) Bem1p-TAP was isolated by tandem immunoprecipitations using IgG and calmodulin beads. The IgGimmunoprecipitated material was treated with TEV protease as indicated, before immunoprecipitations with calmodulin beads. Immunoblotting was done using the indicated antibodies. $(C)$ The immunoprecipitated samples from Bemlp-TAP cell extracts were treated as in $B$ and also with $\lambda$-phosphatase ( $\lambda$-PPase) and phosphatase inhibitor (PPase-[I]) as indicated. The antibodies used for immunobloting are shown on the right, and the relative ratios of the signal intensities are shown at the bottom. (D) Extracts from $C D C 28^{+}$and cdc28-as1 cells carrying cMyc-tagged Bem1p on a lowcopy plasmid (plasmid pDLB2226) (see Table 2) were prepared using a urea extraction buffer and analyzed by SDS PAGE and immunoblotting with an anti-Myc antibody. The cells were treated with DMSO alone or 1 NM-PP1 (at $5 \mu \mathrm{M})$ for the indicated times. (E) Vacuole fusion activity using cytosol from cdc28-as1 cells. DMSO alone or $1 \mathrm{NM}$-PP1 (at $5 \mu \mathrm{M}$ for $15 \mathrm{~min}$ ) was added as indicated. Fusion was evaluated colorimetrically and the average and standard deviation of the relative fusion activities from at least three independent experiments is shown.

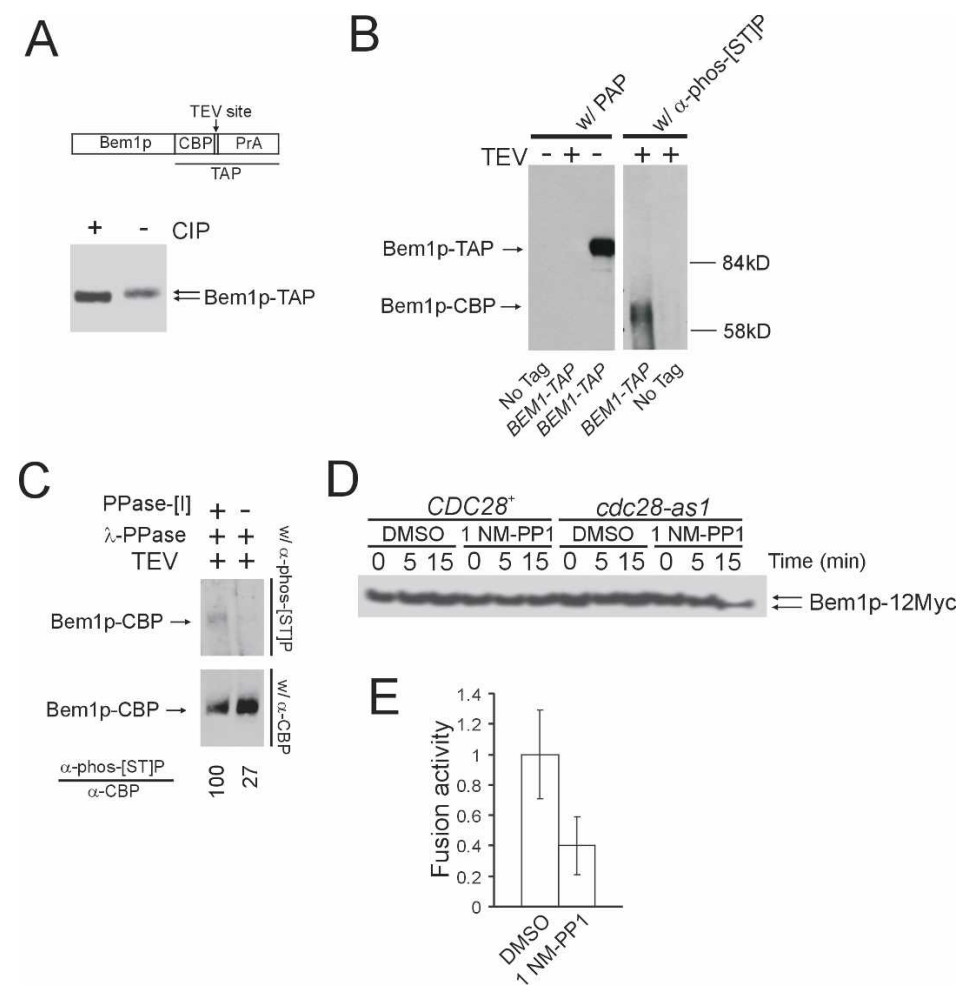

mation is available (Fig. 5A). Not surprisingly, all the sites are conserved in the sensu stricto Saccharomyces species (S. mikatae, S. kudriavzevii, S. paradoxus, and $S$. bayanus). S. castellii is a more distantly related sensu lato Saccharomyces species, and alignment of Bemlp to its ortholog in this species is likely to be more useful (Cliften et al. 2003). In S. castellii only positions 26 and 72 are conserved, while in the two Candida species positions 51 and 72 are conserved (Fig. 5A). The extreme $N$ terminus of the $C$. albicans Bemlp does not show significant conservation with the $S$. cerevisiae Bem 1p, and the highlighted position may not correspond to Ser51. Overall, the only conserved site in all of these cases is Ser72 (Fig. 5A), which is a preferred [ST]-P-X-[KR] Cdk consensus site. Ser72 also represents the first amino acid residue of the SH3-1 domain, which spans from position 72 to 132 , based on PROSITE software predictions (Gattiker et al. 2002).

To test whether position 72 might be targeted for Cdkmediated phosphorylation, we replaced Ser72 with Ala (to abolish phosphorylation) or Asp (to mimic phosphorylation) and evaluated the vacuolar morphology of bem $1 \Delta$ or $\operatorname{cln} 3 \Delta$ cells expressing these Bem1p mutants from low-copy plasmids as C-terminal cMyc fusions (Fig. 5B). The S72A and S72D mutants were expressed at levels similar to the wild-type cMyc-tagged Bem1p, and their subcellular localization was indistinguishable from wild type (data not shown). We noticed that introduction of the otherwise wild-type cMyc-tagged Bemlp on a lowcopy centromeric plasmid only partially suppressed the vacuolar fragmentation of bem1s cells (Fig. 5B). The plasmid is clearly functional because it does suppress the bud emergence defect of bem1s cells (see Fig. 7, below; Irazoqui et al. 2003). The lack of strong suppression of the vacuolar fragmentation might be due to the relative underexpression of cMyc-tagged Bem $1 \mathrm{p}$ from this plasmid compared with endogenous levels (Irazoqui et al. 2003). Consistent with this interpretation, cMyc-tagged Bem 1p expressed from a high-copy $(2 \mu)$ plasmid at levels no more than twofold higher compared with endogenous Bem1p (Irazoqui et al. 2003) showed strong suppression of the vacuolar fragmentation of bem1s cells (Fig. 8D, below).

Nonetheless, it is important to note that the fact that wild-type cMyc-tagged Bemlp from a low-copy plasmid only partially suppressed vacuolar fragmentation conveniently allows one to evaluate the effects of the S72D substitution even in bem1s (but $C L N 3^{+}$) cells. It was clear that S72D Bem1p suppressed vacuolar fragmentation better than wild-type Bem $1 \mathrm{p}$, and, conversely, the S72A Bem1p mutant did not suppress at all the vacuolar fragmentation of bem1s cells (Fig. 5B,C). Because the S72A mutant is functional in bud emergence (see below, Fig. 7), the lack of suppression in vacuolar fragmentation is unlikely to be due to the production of a nonfunctional unfolded protein. Finally, introduction of the S72D, but not the S72A, Bem 1p mutant in $c \ln 3 \Delta$ cells also significantly suppressed their vacuolar fragmentation (Fig. $5 \mathrm{~B}, \mathrm{Cl}$.

To further test the role of Ser72, we overexpressed 
A

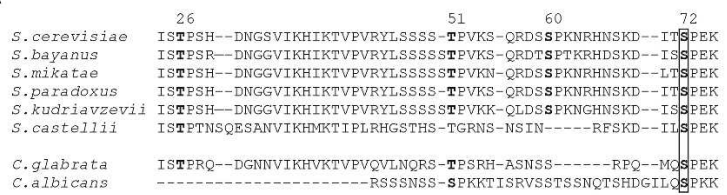

B

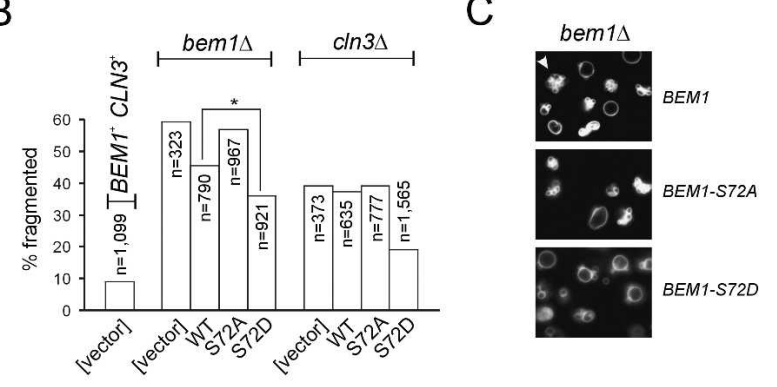

D

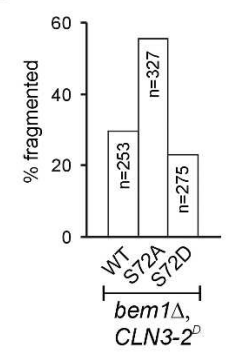

$E$

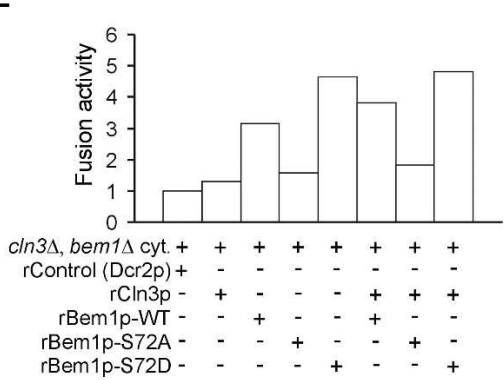

Figure 5. Ser at position 72 in Bem $1 \mathrm{p}$ is critical for vacuolar morphology. (A) Bemlp amino acid sequences (positions 24-75) in Saccharomyces and Candida species. The numbering on top refers to $S$. cerevisiae Bemlp. The amino acids putatively targeted for Cdk-dependent phosphorylation are shown in bold, and the conserved Ser at position 72 is bracketed. (B) Homozygous diploid wild-type (WT), bem1s, or cln3s cells (BY4743 background) were transformed with the low-copy empty vector, wild-type, or mutant cMyc-tagged Bemlp as indicated. The transformants were then stained with FM4-64 to observe their vacuolar morphology by fluorescence microscopy. The percent of cells with vacuolar fragmentation and the number of cells scored in each case $(n)$ is shown. Where indicated with an asterisk, the $P$ value associated with a $\chi^{2}$ significance test was found to be significant $\left(2.7 \times 10^{-14}\right)$. $(C)$ Representative photographs of the indicated strains from $B$. The arrow indicates an example of vacuolar fragmentation. $(D)$ Homozygous diploid bem1s cells (BY4743 background) carrying the $C L N 3-2^{D}$ allele on a low-copy plasmid were also transformed with the low-copy wild-type or mutant cMyc-tagged Bem1p as indicated. Vacuolar morphology was evaluated as in $B$ above. $(E)$ Fusion reactions were performed as described in Materials and Methods, using cytosol from cells lacking both CLN3 and BEM1 (strain SMY01) (Table 1) and recombinant proteins from bacteria as indicated. The average of the relative activities from two independent experiments is shown in each case. The data from the two experiments had a Pearson correlation value of 0.82 , and a paired two-sample $t$-test value of 0.009 .

Cln3p in cells carrying wild-type or Ser72 Bem1p mutants (Fig. 5D). We found that cells carrying both the CLN3-2 ${ }^{D}$ and BEM1-S72A alleles had fragmented vacuolar morphology (Fig. 5D). Thus, the S72A substitution blocks the ability of $\mathrm{Cln} 3 \mathrm{p}$ to promote vacuole fusion in vivo. We then performed in vitro fusion reactions using cytosol from cells lacking both $C \ln 3 p$ and Bem1p, supplemented with various combinations of recombinant Cln3p, Bem1p, Bem1p-S72A, and Bem1p-S72D (Fig. $5 \mathrm{E})$. The highest activity was observed upon addition of Bem1p-S72D with or without $C \ln 3 p$, and the lowest upon addition of Bem1p-S72A. Also, addition of Cln3p alone or with Bem1p-S72A did not increase fusion activity (Fig. 5E). Therefore, these in vitro results completely support our in vivo evidence that $C \ln 3 p$ requires Bem $1 p$ to promote fusion. Furthermore, the S72A substitution in Bemlp blocks fusion, while the S72D mutation promotes it. Interestingly, addition of wild-type Bem 1p also increased fusion activity in the absence of $\mathrm{Cln} 3 \mathrm{p}$, albeit not to the same level as Bem1p-S72D. Thus, it is possible that in the absence of $C \ln 3 p$, when large amounts of exogenous Bem $1 \mathrm{p}$ are added, Bem $1 \mathrm{p}$ can still be modified in these extracts, perhaps by other cyclin/Cdk complexes. Taken together, our results from the in vitro fusion assays are in very good agreement with our in vivo evidence, and suggest that $C \ln 3 p$ and Bem $1 p$ play direct roles in vacuole fusion, with Bemlp acting downstream of $\mathrm{Cln} 3 \mathrm{p}$.

Overall, the evidence supports the notion that the conserved Ser at position 72 in the first SH3 domain of Bem1p might be targeted for Cdk-mediated phosphorylation, and that the phosphorylated form promotes vacuolar homotypic fusion. It is possible that the other sites may also be phosphorylated. However, because the S72D mutant significantly suppressed the vacuolar fragmentation of $c \ln 3 \Delta$ and bem $1 \Delta$ cells in vivo and in vitro, while the S72A mutant did not, it is reasonable to conclude that even if additional sites are phosphorylated, phosphorylation of Ser72 has the most significant biological consequences for vacuolar biogenesis.

We then used 2D gel electrophoresis to better resolve putative Bem 1p isoforms (Fig. 6A). Indeed, an acidic isoform present in wild-type Bem1p (Fig. 6A, top panel) was absent in cells lacking Cln3p (Fig. 6A, middle panel) or in cells carrying Bem1p-S72A (Fig. 6A, bottom panel). We also noticed that Bem1p-S72A migrated slightly faster than wild-type Bem $1 p$ in standard one-dimensional SDSPAGE, similar to wild-type Bem1p in cln3s cells (Fig. $6 \mathrm{~B})$. Together, these results strongly suggest that Bem $1 \mathrm{p}$ is phosphorylated at Ser72 in a Cln3p-dependent manner in vivo.

Finally, we examined whether Bemlp expressed in bacteria could be phosphorylated in vitro. For this experiment we overexpressed CLN3 from a high-copy plasmid carrying epitope-tagged CLN3 under the control of a galactose-inducible promoter. It has been previously shown that overexpression of $C \ln 3 p$ allows for the recovery of detectable Cln3p-associated kinase activity against histone $\mathrm{H1}$, and that most of that activity is Cdc28p-dependent (Tyers et al. 1992, 1993). Indeed, the immunoprecipitated $C \ln 3 p$ had associated kinase activity against recombinant Bem $1 p$, albeit to significantly lower levels compared with histone H1 (Fig. 6C). We also performed similar reactions using commercially avail- 
A

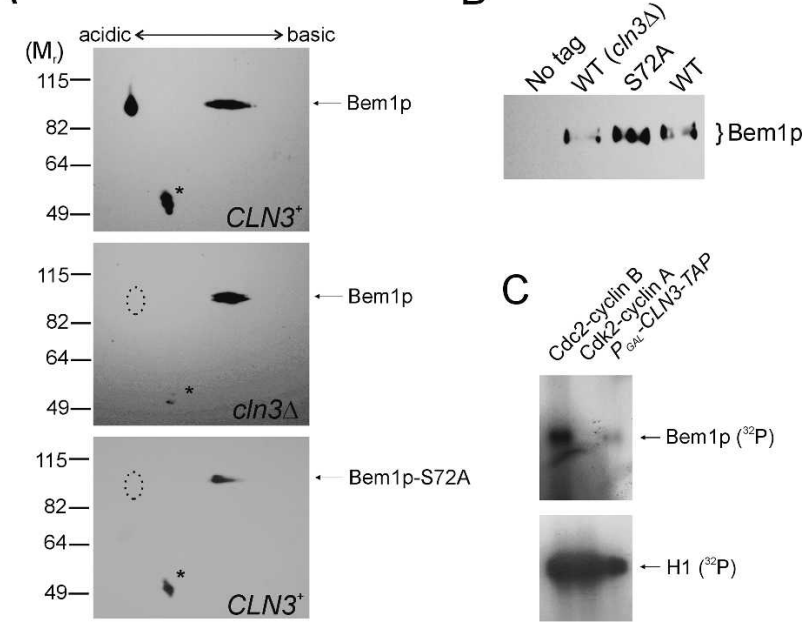

Figure 6. (A) Resolution of Bem1p isoforms by 2D gel electrophoresis. Extracts from cells of the indicated CLN3 genotype were transformed with the low-copy wild-type or mutant cMyctagged Bemlp as indicated and subjected to 2D gel electrophoresis and immunoblotting with an anti-Myc antibody as described in the Materials and Methods. A degradation product is indicated by an asterisk $\left({ }^{\star}\right)$. A broken line circles the location of the acidic full-length isoform absent from the two lower panels. (B) The samples from $A$ were also analyzed by standard onedimensional SDS-PAGE and immunoblotting. (C) Phosphorylation of Bemlp in vitro. (Top) Autoradiogram of kinase reactions using recombinant Bemlp as a substrate and commercial preparations of human $\mathrm{Cdk} /$ cyclin complexes and $\mathrm{Cln} 3 \mathrm{p}$-associated kinase activity from yeast cells, as indicated. Exposure time was 10 d. (Bottom) Autoradiogram of kinase reactions using histone $\mathrm{H} 1$ as a substrate and the same kinase preparations as in the top panel. Exposure time was $4 \mathrm{~h}$.

able preparations of human $\mathrm{Cdc} 2 / \operatorname{cyclinB}$ and $\mathrm{Cdk} 2 /$ cyclinA complexes. Surprisingly, Cdc2/cyclinB apparently phosphorylated Bemlp (Fig. 6C). Whether this reflects "background" nonspecific levels of activity of this preparation is unclear at this point. Note that although the Cdc2 signal appears stronger than the signal from $\mathrm{Cln} 3$ p (Fig. $6 \mathrm{C}$ ), the commercial Cdc2 preparation we used was much more active against histone $\mathrm{H} 1$ than the Cln3p-associated activity we obtained from yeast cells.

\section{Opposing role of Ser72 in bud emergence}

Since Bem1p was originally identified for its role in bud emergence (Bender and Pringle 1991), we next examined whether our Bem1p Ser72 mutants have phenotypes associated with bud emergence. To quantify the bud emergence defect we measured the frequency of unbudded cells containing spindles. The S72A mutant was fully functional in bud emergence (Fig. 7), in contrast to its total lack of complementing activity in vacuole fusion (Fig. 5). Interestingly, the S72D substitution did not fully complement the bud emergence defect of bem1s cells (Fig. 7). Thus, while phosphorylation of Ser72 promotes vacuole fusion, it is not required for and it might even negatively affect bud emergence.
Overexpression of $C d c 42 p$ or $C d c 24 p$ suppresses the vacuolar fragmentation of $\operatorname{cln} 3 \Delta$ cells

We next examined whether Cln3p's requirement for vacuolar homotypic fusion might reflect perturbations of Cdc42p activity, since Bem $1 p$ is a well-established regulator of $\mathrm{Cdc} 42 \mathrm{p}$, and $\mathrm{Cdc} 42 \mathrm{p}$ is also required for the docking step of vacuolar homotypic fusion (Eitzen et al. 2001; Muller et al. 2001). To perhaps bypass the requirement for $C \ln 3 p$, we activated the $C d c 42 p$ GTPase cycle by overexpressing wild-type $C D C 42$, or its exchange factor $C D C 24$, in $c \ln 3 \Delta$ cells and evaluated their vacuolar morphology. Remarkably, overexpression of CDC42 or CDC24 completely suppressed the fragmented vacuolar morphology of $c \ln 3 \Delta$ cells (Fig. 8A,B). On the other hand, overexpression of CDC42 did not significantly suppress the vacuolar fragmentation of bem1s cells (Fig. 8B). $C D C 24$ overexpression weakly suppressed the vacuolar fragmentation in bem1s cells (Fig. 8B). Overall, overexpression of Cdc42p, in the presence of Bemlp, is sufficient to bypass the requirement for $C \ln 3 p$ in vacuole fusion.

Furthermore, in the in vitro fusion assay, addition of Cdc42p did not rescue the fusion defect of bem1s cytosolic extracts (Fig. 8C), suggesting again that Bemlp is required for $\mathrm{Cdc} 42 \mathrm{p}$ to promote fusion. Interestingly, when the extracts were supplemented with Bem1p-S72A together with Cdc42p there was an increase in fusion activity $(-60 \%)$ (Fig. $8 \mathrm{C}$ ), but not to the same extent as when the extracts were supplemented with Bem1p-S72D (see Fig. 5E).

We next examined several other Bem1p amino acid substitutions at positions that are important for Bem 1p's biological roles (Irazoqui et al. 2003) for their ability to suppress vacuolar fragmentation of bem1s cells (Fig. 8D). For example, a P208L substitution affects interactions with various effectors and scaffolds, P355A inter-

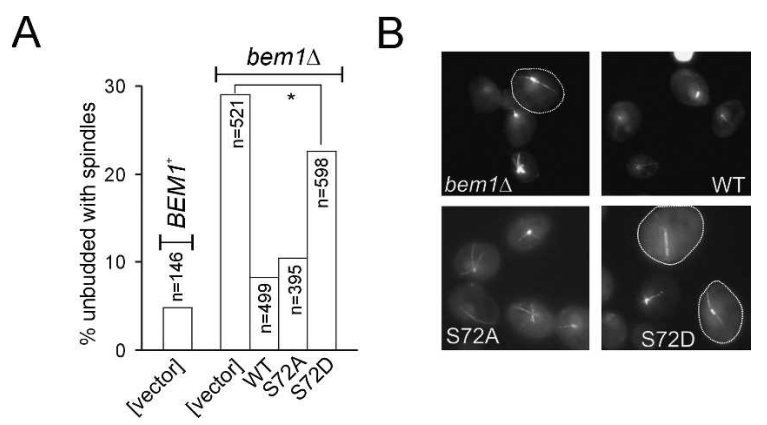

Figure 7. Homozygous diploid wild-type (WT) or bem1s cells (BY4743 background) were transformed with the low-copy empty vector, wild-type, or mutant cMyc-tagged Bemlp as indicated. The cells were grown at $37^{\circ} \mathrm{C}$, fixed, and processed for immunofluorescence to visualize tubulin. (A) The percent of unbudded cells with spindles and the number of cells scored in each case $(n)$ is shown. Where indicated with an asterisk, the $P$ value associated with a $\chi^{2}$ significance test was found to be significant (0.017). (B) Representative photographs of the indicated strains from $A$. Examples of unbudded cells with spindles are outlined. 
A

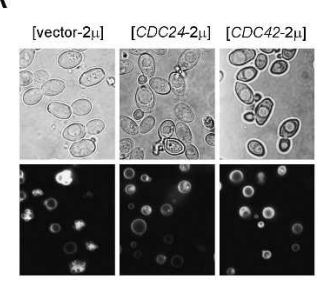

C

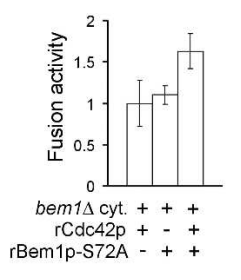

$E$

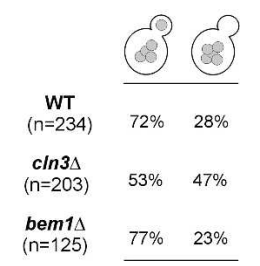

Figure 8. CDC42 or $C D C 24$ overexpression suppresses the vacuolar fragmentation of $c \ln 3 \Delta$ cells. (A) Diploid $c \ln 3 \Delta$ cells (BY4743 background) were transformed with high-copy plasmids as indicated. The transformants were then stained with FM4-64 to observe their vacuolar morphology and photographed through phase optics (top panels) and by fluorescence (bottom panels). (B) The same analysis as in $A$ was also done for bem1 and wild-type (WT) cells, and the percent of cells with vacuolar fragmentation and the number of cells scored in each case $(n)$ is shown. $(C)$ Fusion reactions were performed as described in Materials and Methods, using cytosol from bem $1 \Delta$ cells and recombinant proteins from bacteria as indicated. Fusion was evaluated colorimetrically and the average and standard deviation of the relative fusion activities from at least three independent experiments is shown. (D) Homozygous diploid bem1s cells (BY4743 background) were transformed with the empty high-copy vector or carrying wild-type or mutant cMyc-tagged Bemlp as indicated. The transformants were then stained with FM4-64 to observe their vacuolar morphology by fluorescence microscopy. The percent of cells with vacuolar fragmentation and the number of cells scored in each case $(n)$ is shown. $(E)$ Cells lacking Bem1p are not defective in vacuolar segregation. Exponentially growing cells of the indicated genotype were transferred in medium containing FM4-64 for $1 \mathrm{~h}$, washed, resuspended in fresh medium, and allowed to grow for another $4 \mathrm{~h}$ before they were examined microscopically. The number of cells examined is shown in parentheses. The scored cells had a bud diameter $\leq 0.4$ of the mother cell's diameter. The percentage of cells with the indicated vacuolar morphology is shown.

feres with a postulated conformational change of Bemlp, R369A inhibits interactions with phosphoinositides, and K482A affects interaction with Cdc24p (Irazoqui et al. 2003). The R369A substitution did not have any effect (Fig. 8D), suggesting perhaps that lipid interactions are not important for Bemlp's function in vacuolar homo- typic fusion. To varying degrees, the other Bemlp mutants also significantly suppressed vacuolar fragmentation of bem1s cells (Fig. 8D). Notably, the weakest suppression, albeit still significant, was observed with the P208L substitution (Fig. 8D), which affects proteinprotein interactions through the SH3-2 domain (Irazoqui et al. 2003).

Finally, since the actin cytoskeleton plays a major role in vesicle transport in the bud and vacuole inheritance (Weisman 2003; Pruyne et al. 2004), we decided to evaluate vacuolar segregation in bem1s cells. When we followed the "old" vacuolar compartment synthesized in previous cell divisions, we found that bem $1 \Delta$ and wildtype cells equally distribute their vacuoles between the mother and the bud (Fig. 8E), unlike cln3 $\Delta$ cells, which have a weak but measurable defect (Fig. 8E; Han et al. 2003). Furthermore, overexpression of CDC42 did not alter the vacuolar segregation of $\operatorname{cln} 3 \Delta$ cells (data not shown). Overall, these results suggest that it is unlikely that the mechanism we describe here linking $C \ln 3 p$ and Bem $1 p$ significantly affects vacuole segregation.

\section{Discussion}

In this study we present experiments that link the G1 cyclin Cln3p with vacuole fusion, through Bem1p and the Cdc42p GTPase. We discuss our findings in the context of the known roles of these proteins and how they might affect vacuolar homeostasis.

We were initially led to Bem1p because bem $1 \Delta$ cells are large and their vacuolar compartment is also enlarged and fragmented, similar to the situation in $\operatorname{cln} 3 \Delta$ cells (Fig. 2; Han et al. 2003). The experiments we report here suggest that Bemlp is phosphorylated in a Cdk-dependent manner. The biological significance of Ser72 phosphorylation is underscored by the fact that a substitution to Asp that probably mimics phosphorylation suppresses vacuolar fragmentation, while an Ala substitution does not (Fig. 5). Our data strongly point to the link between Cln3p and modification of Ser72 (Fig. 6). In vitro, however, it appears that multiple Cdk complexes can phosphorylate Bem1p. Addition of Bemlp from bacteria can still promote vacuole fusion in vitro, in the absence of $\mathrm{Cln} 3 \mathrm{p}$ (Fig. 5E), suggesting that other cyclin/Cdk complexes might also be able to phosphorylate this exogenous pool of Bem1p. Furthermore, Clb2p/Cdc28p was previously shown to phosphorylate Bemlp (Ubersax et al. 2003) in vitro. This result was further extended in a follow-up study, where Loog and Morgan (2005) found that Clb5p/Cdc28p and Clb2p/Cdc28p phosphorylated Bem $1 p$ with equal efficiency. Nonetheless, in living cells Cln3p certainly plays a unique role in vacuolar biogenesis because loss of any other cyclin does not lead to vacuolar fragmentation (Seeley et al. 2002; Han et al. 2003).

While it is thought that overall vacuolar biogenesis is the result of reciprocal control between vacuole fusion and fission (Peters et al. 2004), there is no evidence that either of these processes oscillate in the cell cycle. Vacuolar inheritance is thought to be coordinated with the 
cell cycle and we have previously reported that loss of $\mathrm{Cln} 3 \mathrm{p}$ leads to mild defects in vacuolar segregation (Fig. 8E; Han et al. 2003), but there are no such defects in bem1 $1 \Delta$ cells (Fig. 8E). Thus, although the mechanism we describe here linking Cln3p with Bem1p certainly impacts on the "steady-state" overall vacuolar homeostasis, it may not necessarily impart an oscillatory feature to it.

On the other hand, Cln3p's vacuolar roles strongly affect the cell size phenotypes associated with CLN3 mutations (see Fig. 3; Han et al. 2003). As we have discussed previously (Han et al. 2003), this does not extend to all cell size mutants. Vacuolar enlargement is usually accompanied by cellular enlargement (Efe et al. 2005) but the converse is not necessarily true and not all large cell size mutants have large vacuoles. This is exemplified in double mutant $c \ln 1,2 \Delta$ cells, which are large overall although they have small vacuoles (Han et al. 2003). A general correlation between vacuolar fragmentation and cell size also does not seem to exist (Fig. 2A). All these observations suggest that Cln3p's role in vacuole fusion is separate from and probably does not impact on the other function of $\mathrm{Cln} 3 \mathrm{p}$ in accelerating initiation of DNA replication.

How might cyclin-dependent phosphorylation of Bem $1 p$ impact on vacuole fusion? Cdc42p is involved in actin dynamics (Etienne-Manneville 2004; Irazoqui and Lew 2004) and vacuole fusion (Eitzen et al. 2001; Muller et al. 2001). Importantly, the temporal requirement of $\mathrm{Cln} 3 \mathrm{p}$ or $\mathrm{Cdc} 42 \mathrm{p}$ for fusion appears to be similar. Loss of either $\mathrm{Cln} 3 \mathrm{p}$ or $\mathrm{Cdc} 42 \mathrm{p}$ leads to vacuolar fragmentation, but once the docking step of fusion is completed neither Cln3p (Fig. 1) nor Cdc42p (Eitzen et al. 2001; Muller et al. 2001) are required anymore for fusion. While it is clear that noncytoskeletal actin is found on the surface of vacuoles and remodeling of this actin pool is required for vacuole fusion (Eitzen et al. 2002), it is not known how it contributes to fusion. This requirement is distinct from the actin cytoskeleton-dependent processes of vacuole segregation (Weisman 2003; Pruyne et al. 2004). Since we find no evidence for a defect in vacuolar segregation in bem1s cells (Fig. 8D), there is no reason to think that the cyclin-dependent phosphorylation of Bem1p we describe here affects this process. Consequently, our data placing Bem $1 p$ downstream of $C \ln 3 p$ (Figs. 3, 5) and the ability of Cdc42p to suppress vacuole fragmentation in cln3s cells (Fig. 8) probably reflect the noncytoskeletal role of actin for vacuole fusion that has been proposed by the Wickner and Mayer laboratories (Eitzen et al. 2001, 2002; Muller et al. 2001).

Organelle-bound actin could also act as a fusion barrier, which must transiently disassemble for vesicles to dock, while later promoting actin assembly is thought to somehow help the docked vesicles to finally fuse (Eitzen 2003). Based on chemical inhibition experiments, it appears that the actin depolymerizing drug latrunculin $\mathrm{B}$ inhibits the last fusion step of vacuole homotypic fusion (Eitzen et al. 2001). Interestingly, the F-actin binding and stabilizing drug jasplakinolide inhibited the docking step of fusion only, and not the last latrunculin B-sensitive step (Eitzen et al. 2001). Given that Cln3p (Fig. 1) and Cdc42p (Eitzen et al. 2001; Muller et al. 2001) are not required after the docking step of fusion, the fact that Ser72 of Bem1p has distinct and even opposing roles in vacuole fusion versus bud emergence (Figs. 5, 7), and the fact that other Bem 1p mutants that affect bud emergence do not affect vacuole fusion (Fig. 8), it is possible that the effects we are observing reflect the "actin as a barrier" model of fusion (Eitzen 2003).

Does cyclin-dependent phosphorylation of Bemlp affect other Bem1p-regulated processes? The S72A substitution is incapable of suppressing vacuolar fragmentation in bem1s cells, but it is still fully active in bud emergence (Figs. 5, 7). On the other hand, the S72D substitution fully suppresses vacuole fragmentation, but it only partially suppresses bud emergence (Figs. 5, 7). These results suggest that Bemlp has distinct roles in bud emergence and vacuole fusion. While presumably locking Ser72 to the phosphorylated state (in the S72D mutant) suffices to suppress vacuolar fragmentation (Fig. 5), it might adversely affect bud emergence (Fig. 7).

The notion that Bem $1 p^{\prime}$ s roles in vacuole fusion and bud emergence may be distinct is also suggested by the fact that other Bem1p mutants (for example, the K482A mutant) that were shown to be unable to suppress bud emergence defects (Irazoqui et al. 2003) retain full complementing ability in vacuole fusion (Fig. 8D). Even the P208L mutant, which cannot complement bud emergence (Irazoqui et al. 2003), partially suppressed vacuolar fragmentation (Fig. 8D). The P208L mutant affects interactions between the SH3-2 domain of Bem1p with various effectors, including the Cla4p kinase. Interestingly, loss of Cla4p leads to vacuolar fragmentation (Seeley et al. 2002), and it is possible that interactions mediated by the SH3-2 domain of Bem1p might be important for vacuole fusion, explaining the incomplete suppression of vacuolar fragmentation we got from the P208L mutant (Fig. 8D).

In any case, since the actin remodeling necessary for vacuole fusion is not the same as the rearrangement of the actin cytoskeleton that takes place during bud emergence, probably identical mechanisms/effectors do not operate in both processes. It should also be noted that while $C \ln 1 \mathrm{p}$ and $C \ln 2 \mathrm{p}$ have established roles in bud emergence (Gulli et al. 2000; Bose et al. 2001; Moffat and Andrews 2004), they do not affect vacuole fusion (Han et al. 2003). Finally, although there are no known proteinprotein interactions mediated by the SH3-1 domain of Bem 1p, it is possible that Ser 72 might impact on vacuole fusion through interactions with effectors that have specific roles in fusion. Given the scaffold role of Bem 1p and the fact that addition of $\mathrm{Cdc} 42 \mathrm{p}$ in the presence of the Bem1p-S72A mutant did not promote fusion as efficiently as the Bem1p-S72D mutant alone /cf. Figs. 8C and $5 \mathrm{E}$ ), it is possible that phosphorylation of Ser72 is important for some kind of protein-protein interactions that promote vacuole fusion. Future experiments need to address in full detail the mechanisms that account for the differences between Bemlp's roles in vacuole fusion and bud emergence. 


\section{Materials and methods}

Strains and DNAs

Cell cultivation, media, and yeast molecular biology techniques were performed as described by Kaiser et al. (1994), unless otherwise indicated. The strains and plasmids used in this study are listed in Tables 1 and 2, respectively. To generate the bem1s cln3s strain (SMY01) we disrupted one CLN3 copy in diploid bem1s cells as described previously (Polymenis and Schmidt 1997). The resulting heterozygote was sporulated, and the segregants were obtained by random spore analysis (Kaiser et al. 1994). All the single cyclin deletions and the bem $1 \Delta$ strain in the BY4741 background were obtained by sporulation of the corresponding homozygous diploid deletion strains distributed from Research Genetics. The phenotypes reported for each strain were obtained after examining several independent transformants or segregants for the strain in question.

The putative phosphorylation site amino acid substitutions were introduced in the BEM1-12MYC low-copy centromeric plasmid pDLB2226 (Irazoqui et al. 2003). We first PCR amplified $B E M 1$ sequences from pDLB2226 using forward primers that encoded the desired mutation (BEM1-S72A-FWD, 5'-CCAAAA AACAGACATAATTCTAAAGATATTACTGCTCCAGAGA AAGTTATAAAAGCCAAATAC-3'; BEM1-S72D-FWD, 5' -CC AAAAAACAGACATAATTCTAAAGATATTACTGATCCAG AGAAAGTTATAAAAGCCAAATAC-3'), and a reverse primer corresponding to sequences up to position +574 of the BEM1 ORF [BEM1-(+574)-REV, 5'-CGACCAATTGGCTTAGCAAT GAACC- $3^{\prime}$ ]. The PCR products were purified by agarose gel electrophoresis and used as primers in a second PCR reaction with pDLB2226 as template and a forward primer corresponding to BEM1 sequences up to position -186 [BEM1-(-186)-FWD,

Table 1. Strains used in this study

\begin{tabular}{|c|c|c|}
\hline Strain & Relevant genotype & Source \\
\hline BY4741 & 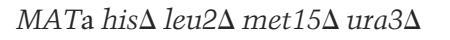 & Res. Genetics \\
\hline BY4742 & 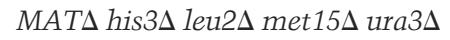 & Res. Genetics \\
\hline BY4743 & BY4741/BY4742 & Res. Genetics \\
\hline W303a & $\begin{array}{l}\text { MATa ade2 trp1 leu2 his3 } \\
\text { ura3 can1 }\end{array}$ & B. Futcher \\
\hline GT108 & $\begin{array}{l}\text { CLN3-3HA:: URA3 (W303a } \\
\text { otherwise) }\end{array}$ & B. Futcher \\
\hline 7503198 & $\begin{array}{l}\text { CLN2-TAP::his3MX (BY4741 } \\
\text { otherwise) }\end{array}$ & Open Biosystems \\
\hline 7499374 & $\begin{array}{l}\text { BEM1-TAP::his3MX (BY4741 } \\
\text { otherwise) }\end{array}$ & Open Biosystems \\
\hline 33340 & $\begin{array}{l}\text { bem } 1 \Delta:: \text { kanMX/bem } 1 \Delta:: \text { kanMX } \\
\text { (BY4743 otherwise) }\end{array}$ & Res. Genetics \\
\hline DKY6281 & $\begin{array}{l}\text { MATa lys2 trp1 ura3 his3 leu2 } \\
\text { suc2 pho8s::TRP1 }\end{array}$ & W. Wickner \\
\hline BJ3505 & $\begin{array}{l}\text { MATa } 1 \text { sy2 trp1 ura3 his3 gal2 } \\
\text { can prb1 pep } 4 \Delta:: \text { HIS3 }\end{array}$ & W. Wickner \\
\hline 13340 & $\begin{array}{l}\text { bem1 } 1 \Delta: \text { : kanMX (BY4742 } \\
\text { otherwise) }\end{array}$ & Res. Genetics \\
\hline SMY01 & $\begin{array}{l}\text { bem1 } 1 \Delta:: \text { KanMX cln } 3 \Delta:: U R A 3 \\
\text { (BY4741 otherwise) }\end{array}$ & This study \\
\hline 30366 & $\begin{array}{l}c \ln 3 \Delta:: k a n M X / c \ln 3 \Delta:: \operatorname{kan} M X \\
\text { (BY4743 otherwise) }\end{array}$ & Res. Genetics \\
\hline 366 & $\begin{array}{l}\operatorname{cln} 3 \Delta:: \operatorname{kan} M X(\mathrm{BY} 4741 \\
\text { otherwise) }\end{array}$ & Res. Genetics \\
\hline DOM90 & bar1::HisG (W303a otherwise) & D. Morgan \\
\hline DOM30 & $\begin{array}{l}\text { cdc28::cdc28-as1 (DOM90 } \\
\text { otherwise) }\end{array}$ & D. Morgan \\
\hline
\end{tabular}

5'-ATTACCCTAAACGGACAAATG-3']. The PCR product of this reaction was also purified by agarose gel electrophoresis and cotransformed into yeast cells together with plasmid pDLB2226, which was previously linearized with SmaI and HindIII digestion (cutting at positions -166 to +330 of the BEM1 ORF, respectively). The gap-repaired plasmid derivatives were then recovered from yeast transformants by standard methods (Kaiser et al. 1994).

To construct plasmids for bacterial expression, we digested the BG1805-based plasmids (purchased from Open Biosystems; see Table 2) with AgeI, which cuts once in the GAL1 promoter all the BG1805 plasmids used here. The digested plasmids were then transformed into a Ura ${ }^{-}$yeast strain for gap repair together with an oligonucleotide that encodes the arabinose $P_{B A D}$ promoter and ribosome-binding site (Guzman et al. 1995) (GAL1PBAD-FWD， 5'-CGGGAACGGATTAGAAGCCGCCGAGCG GGTGACGCTTTTTATCGCAACTCTCTACTGTTTCTCCA TACCCGTTTTTTTGGATGGAGTGAATATACCTCTATAC TTTAACGTCAAGGAGAA-3'). GAL1-PBAD-FWD at its $5^{\prime}$ end carries sequences complementary to GAL1 sequences upstream of the AgeI site, and at its $3^{\prime}$ end sequences complementary immediately upstream of the ATG start codon of the ORF in the BG1805 plasmids, which correspond to the bacteriophage $\lambda$ attR1 site.

The mutant plasmid derivatives were sequenced to verify the introduced mutation and the absence of any other mutations at the Genome Technologies Laboratory of Texas A\&M University.

\section{Vacuolar morphology and size}

For microscopic examination of vacuolar membranes the cells were stained with $\mathrm{N}$-(3-triethylammoniumpropyl)-4-(6-[4-diethylamino]phenylhexatrienyl)pyridinium dibromide, FM4-64, from Molecular Probes, as described by Han et al. (2003), and then examined microscopically with a Nikon Eclipse TS100 inverted fluorescence microscope.

Vacuolar size was evaluated by flow cytometry after staining with the vital vacuolar stain $5^{\prime}$ (and $6^{\prime}$ )-carboxy-2',7'-dichlorofluorescein diacetate, CDCFDA, from Molecular Probes as described previously (Han et al. 2003).

The mean cell volume of live unfixed samples was measured using a Beckman Coulter Z2 Channelyzer. The data were analyzed using the manufacturer's AccuComp software. The geometric mean is indicated in each case.

\section{In vitro vacuole fusion}

Cytosol and vacuoles were prepared and used as described previously (Mayer et al. 1996; Han et al. 2003). Briefly, to measure fusion activity the reaction mixture was equally split, and to each aliquot the same volume of buffer with inhibitor (or antibody) was added. Buffer-added aliquots were incubated at $27^{\circ} \mathrm{C}$ or on ice, the fusion activities of which were measured after 90 min and set as $100 \%$ or $0 \%$ fusion activity, respectively. The inhibitor-added aliquot was incubated at $27^{\circ} \mathrm{C}$ for the same time and its fusion activity was measured to evaluate the relative fusion activity.

\section{Protein analysis}

Unless otherwise indicated, the gels for SDS-PAGE (Laemmli 1970) contained $8 \%$ of a $29: 1$ acrylamide/bis-acrylamide solution. Immunoprecipitations and TEV cleavage of TAP-tagged Bem $1 \mathrm{p}$ were performed according to the protocols and reagents recommended by Rigaut et al. (1999). Briefly, $5 \times 10^{9}$ cells of 
Table 2. Plasmids used in this study

\begin{tabular}{|c|c|c|}
\hline Plasmid & Relevant characteristic & Source \\
\hline p205 & CEN $\left[C L N 3-2^{D}\right]$ URA3 & F. Cross \\
\hline pDLB2226 & CEN [BEM1-12MYC] LEU2 & D. Lew \\
\hline pDLB2226-S72A & CEN [BEM1-S72A-12MYC] LEU2 & This study \\
\hline pDLB2226-S72D & CEN [BEM1-S72D-12MYC] LEU2 & This study \\
\hline pTH113 & $2 \mu[C D C 24] L E U 2$ & T. Höfken \\
\hline pTH114 & $2 \mu[C D C 42] L E U 2$ & T. Höfken \\
\hline pDLB2374 & $2 \mu[B E M 1-12 M Y C] L E U 2$ & D. Lew \\
\hline pDLB2375 & $2 \mu[B E M 1-P 208 L-12 M Y C]$ LEU2 & D. Lew \\
\hline pDLB2377 & $2 \mu[B E M 1-P 355 A-12 M Y C] L E U 2$ & D. Lew \\
\hline pDLB2378 & $2 \mu[B E M 1-R 369 A-12 M Y C]$ LEU2 & D. Lew \\
\hline pDLB2379 & $2 \mu[B E M 1-K 482 A-12 M Y C]$ LEU2 & D. Lew \\
\hline pBAD-DCR2 & {$\left[P_{B A D}-D C R 2-T A G\right]$} & This study \\
\hline pBAD-CLN3 & {$\left[P_{B A D^{-}} C L N 3-T A G\right]$} & This study \\
\hline pBAD-BEM1 & {$\left[P_{\left.B A D^{-}-B E M 1-T A G\right]}\right.$} & This study \\
\hline pBAD-BEM1-S72A & {$\left[P_{\left.B A D^{-B E M 1-S 72 A-T A G]}\right]}\right.$} & This study \\
\hline pBAD-BEM1-S72D & {$\left[P_{B A D}-B E M 1-S 72 D-T A G\right]$} & This study \\
\hline pBAD-CDC42 & {$\left[P_{B A D}-C D C 42-T A G\right]$} & This study \\
\hline BG1805-DCR2 & $2 \mu\left[P_{G A L 1}-D C R 2-T A G\right] U R A 3$ & Open Biosystems \\
\hline BG1805-CLN3 & $2 \mu\left[P_{G A L 1}-C L N 3-T A G\right] U R A 3$ & Open Biosystems \\
\hline BG1805-BEM1 & $2 \mu\left[P_{G A L 1}-B E M 1-T A G\right] U R A 3$ & Open Biosystems \\
\hline BG1805-CDC42 & $2 \mu\left[P_{G A L 1}-C D C 42-T A G\right] U R A 3$ & Open Biosystems \\
\hline
\end{tabular}

untagged or TAP-tagged BEM1 cells from the exponential phase of growth were used. Cell extracts were prepared in the presence of protease and phosphatase inhibitor cocktails (Sigma). After the first immunoprecipitation with IgG-agarose beads, the beads were washed twice with RIPA buffer and once with TEV reaction buffer. Eighty units of TEV for $30 \mathrm{~min}$ at $30^{\circ} \mathrm{C}$ were used to cleave the protein A domain. The phosphatase experiment shown in Figure 4A was done as described previously (Liakopoulos et al. 2003). For the phosphatase experiments in Figure 4C, Bem1p-CBP on calmodulin beads was washed three times with RIPA buffer and once with $\lambda$-phosphatase reaction buffer. Then $500 \mathrm{U}$ of $\lambda$-phosphatase and $10 \times$ of protease inhibitor cocktail were added and incubated at $30^{\circ} \mathrm{C}$ for $30 \mathrm{~min}$ in the presence or absence of $10 \times$ phosphatase inhibitor cocktail.

For protein surveillance, cell extracts were prepared using a urea extraction buffer as described by Ubersax et al. (2003). The differences in Bemlp's electrophoretic mobility in 1D SDSPAGE are better resolved if the samples are desalted prior to electrophoresis (using a kit from Pierce, according to their instructions), and electrophoresis is performed at a constant voltage of $70 \mathrm{~V}$ (instead of the typical $200 \mathrm{~V}$ applied to minigels).

Two-dimensional gel electrophoresis was performed using carrier ampholytes (pH 4-6; GE Healthcare) in acrylamide tubes according to the method of O'Farrell (1975). Seven-centimeterlong tube gels containing $0.5 \%$ ampholytes were focused for $\sim 1000$ volt hours without prefocusing. The tubes were extruded and soaked, sequentially, in SDS sample buffer containing dithiothreitol or iodoacetamide. The tube gels containing the reduced and alkylated proteins were subsequently placed on top of $8 \%$ polyacrylamide slab minigels and subjected to SDS PAGE (Laemmli 1970). Prior to immunostaining, the proteins were electroblotted onto PVDF membranes (Immobilon $\mathrm{P}^{\mathrm{sq}}$; Millipore) at $100-200 \mathrm{~mA}$ overnight using $10 \mathrm{mM}$ CAPS, $10 \%$ methanol ( $\mathrm{pH} 11)$.

For immunostaining, protein A fusion proteins were detected with the peroxidase-anti-peroxidase (PAP) soluble complex reagent from Sigma. The anti-Pgk1p antibody was from Molecular Probes. The anti-phospho-[ST]P, anti-CBP, anti-HA, and antiMyc antibodies were from Abcam. The horseradish peroxidase- conjugated secondary antibodies used for immunoblotting were also from Abcam. All antibodies were used at the dilutions recommended by the manufacturers. The blots were processed with reagents from Pierce.

\section{Bacterial expression}

The pBAD-based plasmids were transformed into Escherichia coli (strain XL1-Blue). For protein expression we followed previously published procedures (Guzman et al. 1995). From 50$100-\mathrm{mL}$ cultures we obtained soluble protein from all the constructs we describe here. The exception was $C \ln 3 p$, which required larger cultures $(0.51-1 \mathrm{~L})$ because the majority of the recombinant protein appeared to be insoluble. We purified the recombinant proteins through their $6 \mathrm{xHis}$ epitope, using TALON $\mathrm{Co}^{2+}$ affinity beads (BD Biosciences), according to the manufacturer's instructions.

\section{Kinase assays}

Human Cdc2/cyclin B and Cdk2/cyclin A were from New England Biolabs, and used at $5 \mathrm{U}$ per reaction. $\mathrm{Cln} 3 \mathrm{p}$-associated activity was obtained from yeast cells (strain W303a) carrying plasmid BG1805-CLN3 (see Table 2), using TALON Co ${ }^{2+}$ affinity beads (BD Biosciences), according to the manufacturer's instructions. The Cln3p-associated activity was from $\sim 10^{10}$ cells initially grown in raffinose, but $4 \mathrm{~h}$ prior to harvesting, the culture was induced with $2 \%$ galactose. Histone $\mathrm{H} 1$ was from Sigma and used as a substrate at $5 \mu \mathrm{g}$ per reaction. Bemlp was obtained from bacteria as described above. The reactions also contained $5 \mu \mathrm{Ci}\left[\gamma_{-}{ }^{32} \mathrm{P}\right] \mathrm{ATP}, 100 \mu \mathrm{M}$ ATP, $50 \mathrm{mM}$ Tris- $\mathrm{HCl}(\mathrm{pH}$ 7.5), $10 \mathrm{mM} \mathrm{MgCl}$, $1 \mathrm{mM}$ EGTA, $2 \mathrm{mM}$ DTT, and $0.01 \%$ Brij 35. All the reactions ( $30 \mu \mathrm{L}$ total volume) were performed at room temperature for $20 \mathrm{~min}$.

\section{Immunofluorescence microscopy}

Unless otherwise indicated, we followed the protocols of the Botstein laboratory as described at http://genome-www. 
stanford.edu/group/botlab. DAPI, 4'6-diamidino-2-phenylindole, was from Sigma. The anti-tubulin primary antibody and the secondary FITC-conjugated antibody were from Abcam. For the experiment shown in Figure 7, the cells were grown at $37^{\circ} \mathrm{C}$ to exacerbate the bud emergence defect of bem1s cells. The samples were examined with a Nikon Eclipse TS100 inverted fluorescence microscope.

\section{Acknowledgments}

We thank all the members of the Polymenis laboratory for comments and advice; Elena Lyuksyutova for expert technical assistance with 2D gel electrophoresis; D. Morgan, F. Cross, B. Futcher, T. Höfken, W. Wickner, W. Harper, C. Wittenberg, and D. Lew for generously providing us with reagents; D. Lew, D. Morgan, and C. Wittenberg for useful discussions; and J.C. Hu, M. Kladde, and L. Guarino for help and advice. This work was supported by a grant from the National Institutes of Health (R01-GM062377) to M.P.

\section{References}

Barelle, C.J., Bohula, E.A., Kron, S.J., Wessels, D., Soll, D.R., Schafer, A., Brown, A.J., and Gow, N.A. 2003. Asynchronous cell cycle and asymmetric vacuolar inheritance in true hyphae of Candida albicans. Eukaryot. Cell 2: 398-410.

Bender, A. and Pringle, J.R. 1991. Use of a screen for synthetic lethal and multicopy suppressee mutants to identify two new genes involved in morphogenesis in Saccharomyces cerevisiae. Mol. Cell. Biol. 11: 1295-1305.

Bishop, A.C., Ubersax, J.A., Petsch, D.T., Matheos, D.P., Gray, N.S., Blethrow, J., Shimizu, E., Tsien, J.Z., Schultz, P.G., Rose, M.D., et al. 2000. A chemical switch for inhibitorsensitive alleles of any protein kinase. Nature 407: 395-401.

Bose, I., Irazoqui, J.E., Moskow, J.J., Bardes, E.S., Zyla, T.R., and Lew, D.J. 2001. Assembly of scaffold-mediated complexes containing Cdc42p, the exchange factor Cdc $24 p$, and the effector Cla4p required for cell cycle-regulated phosphorylation of Cdc24p. J. Biol. Chem. 276: 7176-7186.

Cliften, P., Sudarsanam, P., Desikan, A., Fulton, L., Fulton, B., Majors, J., Waterston, R., Cohen, B.A., and Johnston, M. 2003. Finding functional features in Saccharomyces genomes by phylogenetic footprinting. Science 301: 71-76.

Costanzo, M., Nishikawa, J.L., Tang, X., Millman, J.S., Schub, O., Breitkreuz, K., Dewar, D., Rupes, I., Andrews, B., and Tyers, M. 2004. CDK activity antagonizes Whi5, an inhibitor of G1/S transcription in yeast. Cell 117: 899-913.

Cross, F.R. 1988. DAF1, a mutant gene affecting size control, pheromone arrest, and cell cycle kinetics of Saccharomyces cerevisiae. Mol. Cell. Biol. 8: 4675-4684.

de Bruin, R.A., McDonald, W.H., Kalashnikova, T.I., Yates III, J., and Wittenberg, C. 2004. Cln3 activates G1-specific transcription via phosphorylation of the SBF bound repressor Whi5. Cell 117: 887-898.

Dirick, L., Bohm, T., and Nasmyth, K. 1995. Roles and regulation of Cln-Cdc28 kinases at the start of the cell cycle of Saccharomyces cerevisiae. EMBO J. 14: 4803-4813.

Efe, J.A., Botelho, R.J., and Emr, S.D. 2005. The Fab1 phosphatidylinositol kinase pathway in the regulation of vacuole morphology. Curr. Opin. Cell Biol. 17: 402-408.

Eitzen, G. 2003. Actin remodeling to facilitate membrane fusion. Biochim. Biophys. Acta 1641: 175-181.

Eitzen, G., Will, E., Gallwitz, D., Haas, A., and Wickner, W. 2000. Sequential action of two GTPases to promote vacuole docking and fusion. EMBO J. 19: 6713-6720.
Eitzen, G., Thorngren, N., and Wickner, W. 2001. Rholp and Cdc $42 \mathrm{p}$ act after Ypt7p to regulate vacuole docking. EMBO J. 20: $5650-5656$.

Eitzen, G., Wang, L., Thorngren, N., and Wickner, W. 2002. Remodeling of organelle-bound actin is required for yeast vacuole fusion. J. Cell Biol. 158: 669-679.

Etienne-Manneville, S. 2004. Cdc42-The centre of polarity. J. Cell Sci. 117: 1291-1300.

Gattiker, A., Gasteiger, E., and Bairoch, A. 2002. ScanProsite: A reference implementation of a PROSITE scanning tool. Appl. Bioinformatics 1: 107-108.

Gulli, M.P., Jaquenoud, M., Shimada, Y., Niederhauser, G., Wiget, P., and Peter, M. 2000. Phosphorylation of the Cdc42 exchange factor Cdc24 by the PAK-like kinase Cla4 may regulate polarized growth in yeast. Mol. Cell 6: 11551167.

Guzman, L.M., Belin, D., Carson, M.J., and Beckwith, J. 1995. Tight regulation, modulation, and high-level expression by vectors containing the arabinose PBAD promoter. I. Bacteriol. 177: 4121-4130.

Han, B.-K., Aramayo, R., and Polymenis, M. 2003. The G1 cyclin Cln3p controls vacuolar biogenesis in Saccharomyces cerevisiae. Genetics 165: 467-476.

Irazoqui, J.E. and Lew, D.J. 2004. Polarity establishment in yeast. J. Cell Sci. 117: 2169-2171.

Irazoqui, J.E., Gladfelter, A.S., and Lew, D.J. 2003. Scaffoldmediated symmetry breaking by Cdc42p. Nat. Cell Biol. 5: 1062-1070.

Jones, E.W., Webb, G.C., and Hiller, M.A. 1997. Biogenesis and function of the yeast vacuole. In The molecular biology of the yeast Saccharomyces (ed. J.R. Pringle et al.), pp. 363-470. Cold Spring Harbor Laboratory Press, Cold Spring Harbor, NY.

Jorgensen, P., Nishikawa, J.L., Breitkreutz, B.J., and Tyers, M. 2002. Systematic identification of pathways that couple cell growth and division in yeast. Science 297: 395-400.

Kaiser, C., Michaelis, S., and Mitchell, A. 1994. Methods in yeast genetics. Cold Spring Harbor Laboratory Press, Cold Spring Harbor, NY.

Kellis, M., Patterson, N., Endrizzi, M., Birren, B., and Lander, E.S. 2003. Sequencing and comparison of yeast species to identify genes and regulatory elements. Nature 423: 241254.

Laemmli, U.K. 1970. Cleavage of structural proteins during the assembly of the head of bacteriophage T4. Nature 227: 680685.

Leeuw, T., Fourest-Lieuvin, A., Wu, C., Chenevert, J., Clark, K., Whiteway, M., Thomas, D.Y., and Leberer, E. 1995. Pheromone response in yeast: Association of Bem $1 \mathrm{p}$ with proteins of the MAP kinase cascade and actin. Science 270: 1210 1213.

Liakopoulos, D., Kusch, J., Grava, S., Vogel, J., and Barral, Y. 2003. Asymmetric loading of Kar9 onto spindle poles and microtubules ensures proper spindle alignment. Cell 112: 561-574.

Loog, M. and Morgan, D.O. 2005. Cyclin specificity in the phosphorylation of cyclin-dependent kinase substrates. Nature 434: 104-108.

Mayer, A., Wickner, W., and Haas, A. 1996. Sec18p (NSF)-driven release of Sec17p ( $\alpha$-SNAP) can precede docking and fusion of yeast vacuoles. Cell 85: 83-94.

Moffat, J. and Andrews, B. 2004. Late-G1 cyclin-CDK activity is essential for control of cell morphogenesis in budding yeast. Nat. Cell Biol. 6: 59-66.

Muller, O., Johnson, D.I., and Mayer, A. 2001. Cdc42p functions at the docking stage of yeast vacuole membrane fusion. EMBO I. 20: 5657-5665. 
O'Farrell, P.H. 1975. High resolution two-dimensional electrophoresis of proteins. J. Biol. Chem. 250: 4007-4021.

Pathak, R., Bogomolnaya, L.M., Guo, J., and Polymenis, M. 2004. Gid8p (Dcrlp) and Dcr2p function in a common pathway to promote START completion in Saccharomyces cerevisiae. Eukaryot. Cell 3: 1627-1638.

Peters, C., Baars, T.L., Buhler, S., and Mayer, A. 2004. Mutual control of membrane fission and fusion proteins. Cell 119: 667-678.

Polymenis, M. and Schmidt, E.V. 1997. Coupling of cell division to cell growth by translational control of the G1 cyclin CLN3 in yeast. Genes \& Dev. 11: 2522-2531.

Pruyne, D., Legesse-Miller, A., Gao, L., Dong, Y., and Bretscher, A. 2004. Mechanisms of polarized growth and organelle segregation in yeast. Annu. Rev. Cell Dev. Biol. 20: 559-591.

Richardson, H.E., Wittenberg, C., Cross, F., and Reed, S.I. 1989. An essential G1 function for cyclin-like proteins in yeast. Cell 59: 1127-1133.

Richman, T.J., Toenjes, K.A., Morales, S.E., Cole, K.C., Wasserman, B.T., Taylor, C.M., Koster, J.A., Whelihan, M.F., and Johnson, D.I. 2004. Analysis of cell-cycle specific localization of the Rdilp RhoGDI and the structural determinants required for $\mathrm{Cdc} 42 \mathrm{p}$ membrane localization and clustering at sites of polarized growth. Curr. Genet. 45: 339-349.

Rigaut, G., Shevchenko, A., Rutz, B., Wilm, M., Mann, M., and Seraphin, B. 1999. A generic protein purification method for protein complex characterization and proteome exploration. Nat. Biotechnol. 17: 1030-1032.

Roberts, C.J., Raymond, C.K., Yamashiro, C.T., and Stevens, T.H. 1991. Methods for studying the yeast vacuole. Methods Enzymol. 194: 644-661.

Schaefer, J.B. and Breeden, L.L. 2004. RB from a bud's eye view. Cell 117: 849-850.

Seeley, E.S., Kato, M., Margolis, N., Wickner, W., and Eitzen, G. 2002. Genomic analysis of homotypic vacuole fusion. Mol. Biol. Cell 13: 782-794.

Shorter, J. and Warren, G. 2002. Golgi architecture and inheritance. Annu. Rev. Cell Dev. Biol. 18: 379-420.

Spellman, P.T., Sherlock, G., Zhang, M.Q., Iyer, V.R., Anders, K., Eisen, M.B., Brown, P.O., Botstein, D., and Futcher, B. 1998. Comprehensive identification of cell cycle-regulated genes of the yeast Saccharomyces cerevisiae by microarray hybridization. Mol. Biol. Cell 9: 3273-3297.

Tyers, M., Tokiwa, G., Nash, R., and Futcher, B. 1992. The Cln3-Cdc28 kinase complex of $S$. cerevisiae is regulated by proteolysis and phosphorylation. EMBO J. 11: 1773-1784.

Tyers, M., Tokiwa, G., and Futcher, B. 1993. Comparison of the Saccharomyces cerevisiae G1 cyclins: $\mathrm{Cln} 3$ may be an upstream activator of $\mathrm{Cln} 1, \mathrm{Cln} 2$, and other cyclins. EMBO . 12: $1955-1968$.

Ubersax, J.A., Woodbury, E.L., Quang, P.N., Paraz, M., Blethrow, J.D., Shah, K., Shokat, K.M., and Morgan, D.O. 2003. Targets of the cyclin-dependent kinase Cdk1. Nature 425: 859-864.

Warren, G. and Wickner, W. 1996. Organelle inheritance. Cell 84: 395-400.

Weisman, L.S. 2003. Yeast vacuole inheritance and dynamics. Annu. Rev. Genet. 37: 435-460.

Wickner, W. and Haas, A. 2000. Yeast homotypic vacuole fusion: A window on organelle trafficking mechanisms. Annu. Rev. Biochem. 69: 247-275.

Wiemken, A. and Durr, M. 1974. Characterization of amino acid pools in the vacuolar compartment of Saccharomyces cerevisiae. Arch. Microbiol. 101: 45-57.

Zhang, J., Schneider, C., Ottmers, L., Rodriguez, R., Day, A.,
Markwardt, J., and Schneider, B.L. 2002. Genomic scale mutant hunt identifies cell size homeostasis genes in S. cerevisiae. Curr. Biol. 12: 1992-2001. 


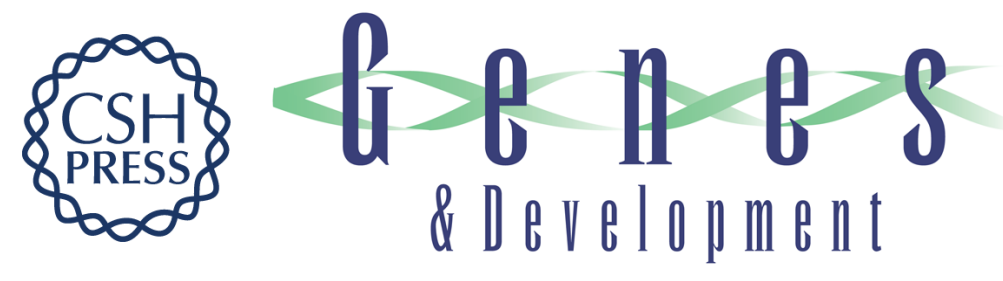

\section{Bem1p, a scaffold signaling protein, mediates cyclin-dependent control of vacuolar homeostasis in Saccharomyces cerevisiae}

Bong-Kwan Han, Lydia M. Bogomolnaya, James M. Totten, et al.

Genes Dev. 2005, 19:

Access the most recent version at doi:10.1101/gad.1361505

References This article cites 51 articles, 20 of which can be accessed free at:

http://genesdev.cshlp.org/content/19/21/2606.full.html\#ref-list-1

License

Email Alerting

Receive free email alerts when new articles cite this article - sign up in the box at the top

Service right corner of the article or click here.

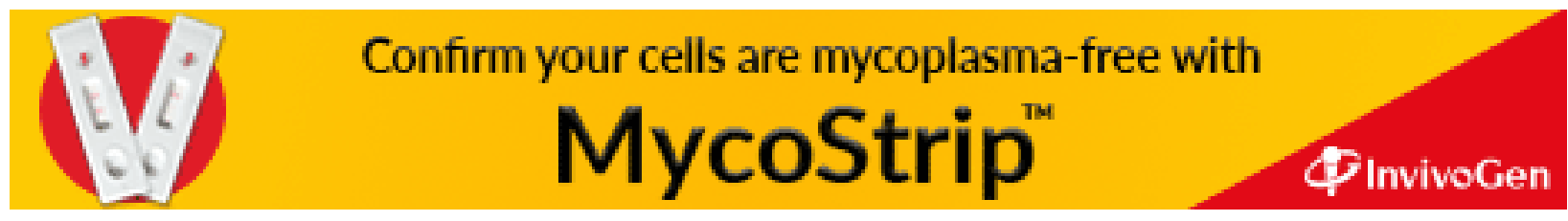

